\title{
A new selective vascular endothelial growth factor receptor 2 inhibitor ablates disease in a mouse model of psoriasis
}

\author{
HENG-XIU YAN, YONG WANG, XIAO-NONG YANG, LI-XIN FU and DONG-MEI TANG \\ College of Life Science and Technology, Southwest University for Nationalities, Chengdu, Sichuan, P.R. China
}

Received April 2, 2013; Accepted May 21, 2013

DOI: $10.3892 / \mathrm{mmr} .2013 .1500$

\begin{abstract}
Psoriasis is a common chronic inflammatory skin disease and its underlying pathogenesis is still not fully understood. Therapeutic interventions are currently limited and restricted to the treatment of symptoms rather than targeting the mechanisms underlying the disease. Vascular remodeling is a hallmark of psoriasis; however, anti-vascular strategies to treat psoriasis have received little attention to date, particularly systemic treatment with a small molecule compound. The aim of this study was to investigate the antiinflammatory effect of a newly identified vascular endothelial growth factor (VEGF) receptor 2 inhibitor, SKLB1002, and its possible mechanism of action in a transgenic mouse model of psoriasis. Fifteen 8-12-week-old K14-VEGF transgenic mice received consecutive intraperitoneal (i.p.) injections of SKLB1002, vehicle or saline for 4 weeks. After 4 weeks of treatment, the disease symptoms were assessed and histological analyses were performed on ear sections by hematoxylin and eosin (HE) and immunohistochemistry staining. Systemic treatment with SKLB1002 reduced symptoms of ear inflammation in K14/VEGF transgenic mice, the pathological score was significantly decreased, and acanthosis, focal parakeratosis, hyperkeratosis and hemangiectasis were improved. Furthermore, systemic treatment with SKLB1002 significantly reduced vascular abnormalities, permeability and T-cell infiltration. These results demonstrated that targeted inhibition of VEGFR2 by a small molecule inhibitor is an effective method, which may be a new therapeutic option for psoriasis therapy.
\end{abstract}

\section{Introduction}

Psoriasis is a chronic inflammatory skin disease that is characterized by erythema, epidermal hyperplasia, inflammatory infiltrates, and enlarged, tortuous and hyperpermeable blood vessels $(1,2)$. Previous studies have shown that the endothelial microvascular bed was increased four-fold in psoriatic skin

Correspondence to: Dr Heng-Xiu Yan, College of Life Science and Technology, Southwest University for Nationalities, no. $16 \mathrm{Yi}$ Huan Lu Nan Si Duan, Chengdu, Sichuan, P.R. China

E-mail: yanhengxiu@hotmail.com

Key words: psoriasis, VEGFR2 inhibitor, target, therapy compared with normal skin (3). Dermal microvascular expansion with abnormal orientation, and dilatation of capillaries in the biopsies of the psoriatic skin revealed that the disease was angiogenesis dependent $(4,5)$ and signified the importance of angiogenesis in psoriasis.

Numerous growth factors and cytokines are involved in angiogenesis. Of all known angiogenic molecules, vascular endothelial growth factor (VEGF) is the key mediator of angiogenesis. Several studies indicate a vital role of VEGF in the pathogenesis of psoriasis: epidermal-derived VEGF is highly upregulated in psoriatic skin lesions (6-8); VEGF serum levels are correlated with disease severity (9); VEGF expression in basal keratinocytes showed psoriatic-like skin inflammation with increased tortuosity and branching of dermal blood vessels in a mouse model $(10,11)$. VEGF receptors 1 and 2 were expressed on the surface of keratinocytes, and the VEGF secreted by the keratinocytes was able to bind to these receptors and activate the signaling pathway in an autocrine manner (12-14). Thus, anti-vascular strategies to treat psoriasis by blocking VEGF binding to VEGFR are anticipated, although to date the majority of anti-angiogenic approaches have primarily focused on the development of cancer therapeutics (15-17).

In the present study, we used a therapeutic approach in a transgenic mouse model (18) of chronic, psoriasis-like skin inflammation, using the anti-VEGFR2 small molecule compound SKLB1002. Systemic treatment of transgenic K14-VEGF mice with SKLB1002 strongly reduced skin inflammation in contrast with control animals. The mice showed a marked improvement in psoriatic phenotype, normalization of the epidermal architecture, and a decrease in the number and size of blood vessels. Furthermore, the immune infiltrate in the skin was reduced in SKLB1002-treated mice.

\section{Materials and methods}

Animals. K14-VEGF transgenic homozygous mice (18) displaying symptoms of human psoriasis were provided by the State Key Laboratory of Biotherapy and Cancer Center, Sichuan University (Sichuan, China). Eight to twelve-week-old mice with moderate to serious psoriasis were selected for the experiment. The mice were housed under specific pathogen-free (SPF) conditions. Animal experiments were approved by the Institutional Animal Care and Use Committee of Southwest University for Nationalities (Chengdu, Sichuan, China). 
SKLB1002. The small molecule compound was donated by $\mathrm{Dr}$ Sheng-Yong Yang from the State Key Laboratory of Biotherapy and Cancer Center, West China Hospital, West China Medical School, Sichuan University (Sichuan, China).

Antibodies. For immunohistochemistry, rabbit anti-mouse antibodies against K6 (Abcam, Cambridge, MA, USA), CD4 (Abcam), CD8 (Abcam), CD31 (Abcam), VEGF (Boster, Fremont, CA, USA), CD54 (Boster) and E-selectin (Boster) were used.

In vivo treatment with the tyrosine kinase inhibitor SKLB1002. K14-VEGF transgenic homozygous mice were divided into three groups as follows: a treatment group receiving SKLB1002 and control groups receiving only vehicle or saline. Mice were dosed with SKLB1002, vehicle or saline once daily by intraperitoneal (i.p.) injection for four weeks. Mice in the treatment group received $50 \mathrm{mg} /$ $\mathrm{kg}$ SKLB1002 (dissolved in 5\% DMSO + 35\% PEG400 suspension, i.p.), which was a dose previously determined to be effective in preparatory experiments (data not shown). Animals in two control groups were treated with the same volume of vehicle (5\% DMSO + 35\% PEG400) and saline, respectively. Twenty-four hours after the final treatment, photographs of mouse ears were obtained before all mice were sacrificed. Ear samples were fixed in $4 \%$ neutral buffered paraformaldehyde for histological analysis.

Histology. Paraffin-embedded skin sections $(\sim 5 \mu \mathrm{m})$ from treated-K14/VEGF mice were stained by $\mathrm{HE}$ and immunohistochemistry. Quantitative assessments of the pathological score, based on Baker's method (19), were performed in five randomly selected high-power fields (x400) in each HE-stained section to assess the severity of psoriasis. The mean epidermal thickness of the ear skin was also measured as an indication of epidermal proliferation. Immunohistochemistry staining was performed according to the antibody protocol, and images were acquired using an Olympus BX60 microscope (Olympus, Japan). Paraffin sections were deparaffinized and rehydrated, then heatinduced antigen retrieval was required for $5 \mathrm{~min}$ prior to immunohistochemical staining. The tissue sections were incubated with the primary antibodies overnight at $4^{\circ} \mathrm{C}$. Cells were counted in five randomly chosen fields (x400) in each immunohistochemically stained section ( $n=5$ for each group). The evaluations were performed by two blinded observers.

Statistical analysis. SPSS 16.0 was used for statistical analysis (SPSS, Inc., Chicago, IL, USA). Data are expressed as the means \pm SD. The statistical analysis in all experiments was performed using one-way analysis of variance (ANOVA) or t-test. $\mathrm{P}<0.05$ was considered to indicate a statistically significant result.

\section{Results}

Systemic treatment with SKLB1002 reduces symptoms of ear inflammation in K14/VEGF transgenic mice. The effects of a newly identified VEGF receptor tyrosine-kinase inhibitor, SKLB1002, were tested in K14/VEGF transgenic mice exhibiting numerous characteristic features of psoriasis. Fifteen 8-12-week-old K14-VEGF transgenic mice received consecutive i.p. injections of SKLB1002, vehicle or saline for 4 weeks. The mice treated with the vehicle or saline exhibited focal skin lesions on their ears, which were highly similar to human psoriasis. By comparison, the mice treated with SKLB1002 showed only hyperaemia and very slight incrassation of the skin on their ears (Fig. 1). SKLB1002 treatment was well-tolerated; the mice showed no signs of sickness and did not lose weight (data not shown).

SKLB1002 treatment normalizes the epidermal architecture in inflamed skin. To better characterize the efficacy of systemic VEGF blockade in reducing psoriasis-like skin inflammation, histological analyses were performed on ear sections obtained from K14/VEGF littermates treated with SKLB1002, vehicle and saline. After 4 weeks of treatment, HE-stained sections revealed the typical histopathological signs of the psoriasis-like phenotype in the control-treated mice, including acanthosis (thickened epidermis), epidermal rete elongation, focal parakeratosis (retention of nuclei in the stratum corneum), hyperkeratosis (thickening of the stratum corneum), hemangiectasis, abundant infiltrated lymphocytes and microabscesses. By contrast, systemic inhibition of VEGF led to a notable reduction in psoriasis-like histological features (Fig. 2).

As shown in Fig. 2, SKLB1002 treatment led to a significant improvement in skin inflammation, which was confirmed by the pathological score for HE-stained preparations based on the Baker score system. The pathological scores of the mice treated with SKLB1002 were significantly different $(n=5$ in each group; $\mathrm{P}<0.01$, one-way ANOVA) from controls. In addition, the average epidermal thickness was reduced by $80 \%$ in the group treated with SKLB1002 when compared with the control groups ( $\mathrm{n}=5$ in each group; $\mathrm{P}<0.01$, one-way ANOVA), whereas the difference between the vehicle-treated group and saline-treated group was not significant. During psoriatic hyperproliferation of keratinocytes, the epidermal hyperproliferation marker keratin 6 displayed a much broader staining pattern. Treatment with SKLB1002 significantly inhibited the expression of keratin 6 in the epidermis. By contrast, keratin 6 expression was marked in the epidermis of vehicle-treated mice. Thus, inhibition of VEGF normalized the epidermal skin architecture in this mouse model of psoriasis.

Decrease in T-cell infiltration mediated by SKLB1002 in vivo. The defining histological features of psoriasis include marked infiltration of $\mathrm{T}$ cells. We thus performed immunohistochemical assays to examine the variations in $\mathrm{CD}^{+}$and $\mathrm{CD}^{+}$ cells in skin sections. As shown in Fig. 3, when compared with the control, SKLB1002 treatment significantly decreased the number of $\mathrm{CD}^{+}$and $\mathrm{CD}^{+}$cells by $71.2 \%$ and $78.5 \%$, respectively, which demonstrated inhibition of the infiltration of inflammatory $\mathrm{T}$ lymphocytes.

SKLB1002 significantly reduces vascular abnormalities and permeability in vivo. As shown in Fig. 4A, when compared with the control, SKLB1002 treatment significantly decreased the expression of VEGF and CD31, which indicated the suppression of the proliferation and dilation of blood vessels. Notably, the average number of blood vessels was significantly 

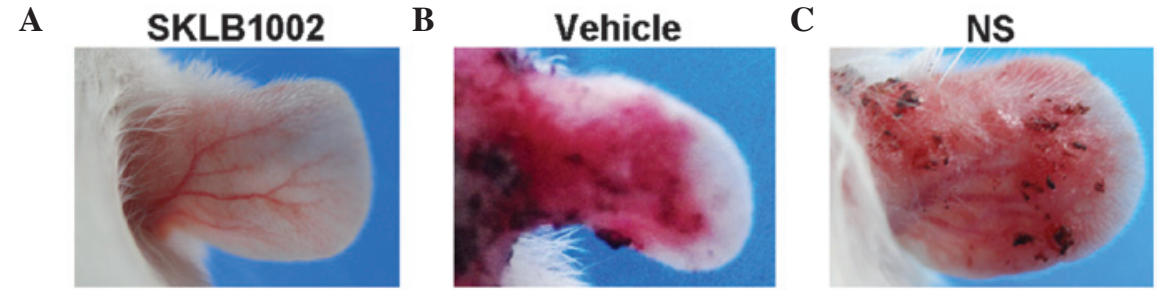

Figure 1. Systemic inhibition of VEGF alleviates the psoriasis-like phenotype in K14-VEGF transgenic mice. Macroscopic characteristics of the ears in the mice after treatment with (A) SKLB1002, (B) vehicle and (C) saline (NS). VEGF, vascular endothelial growth factor.
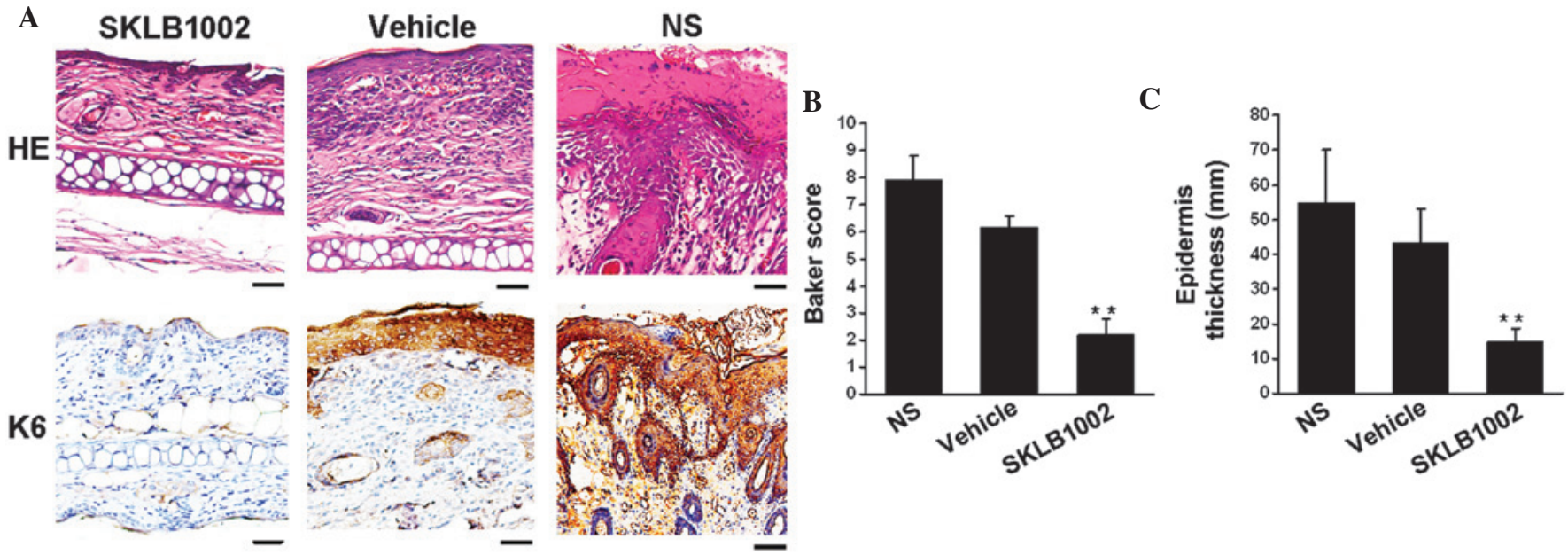

Figure 2. Histological appearance of psoriasis after drug administration in a pharmacodynamic experiment in a mouse model of psoriasis. (A) Images in the upper row show HE staining of tissue sections. Images in the bottom row show the immunohistochemical staining for the epidermal marker K6. Bar, $40 \mu \mathrm{m}$. (B) Quantitative assessments of the pathological score, based on the Baker score system, were performed in 5 randomly chosen high-power fields (means \pm SD, $\mathrm{n}=5$ per group). (C) Average epidermal thickness (means $\pm \mathrm{SD}, \mathrm{n}=5$ per group). Statistical analysis was conducted using a one-way ANOVA test. $\mathrm{P}<0.05$ was considered to indicate a statistically significant result. ${ }^{* *} \mathrm{P}<0.01$. HE, hematoxylin and eosin.

A

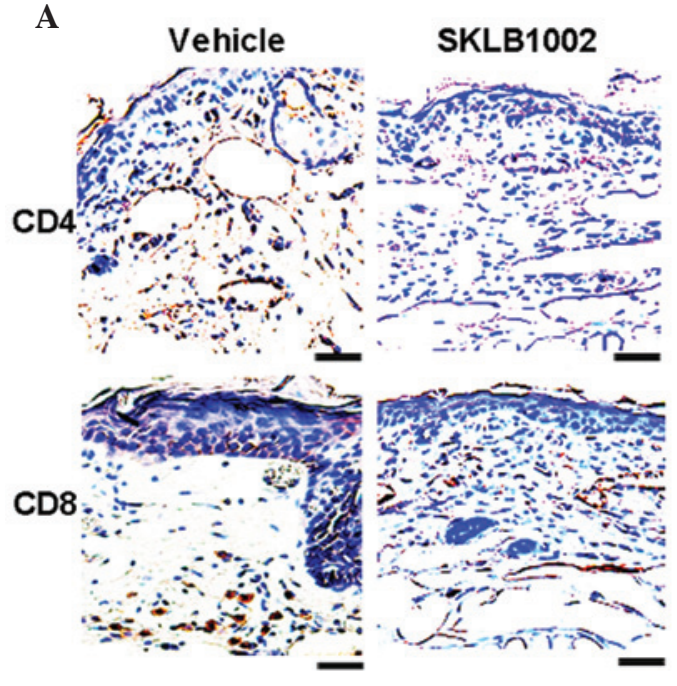

B

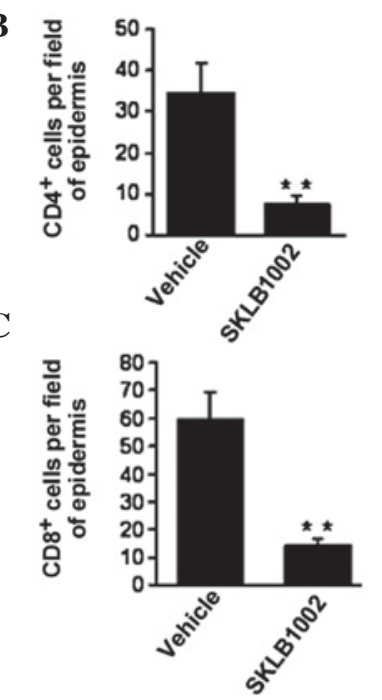

Figure 3. Inhibition of T lymphocytes by SKLB1002 treatment in vivo. (A) Immunohistochemical staining for CD4 and CD8 in tissue sections of vehicle-treated mice and SKLB1002-treated mice. Bar, $40 \mu \mathrm{m}$. The mean number of total (B) $\mathrm{CD}^{+}$and (C) $\mathrm{CD} 8^{+}$cells per field (means $\pm \mathrm{SD}$, $\mathrm{n}=5$ per group) are shown in the bar graphs. ${ }^{* *} \mathrm{P}<0.01$.

reduced $(\mathrm{P}<0.01)$ and the average size of blood vessels $(\mathrm{P}<0.01)$ was significantly smaller in SKLB1002-treated mice than in vehicle control-treated animals.

In particular, the expression of specific endothelial cell adhesion molecules is a hallmark of the hyperplastic and inflamed vessels observed in human psoriatic skin lesions, including the expression of E-selectin, VCAM-1 (CD106) and ICAM-1 (CD54). Compared with the vehicle treatment group, SKLB1002 treatment significantly inhibited the expression of E-selectin, CD54 and CD106 (Fig. 4B). Thus, inhibition of 
A
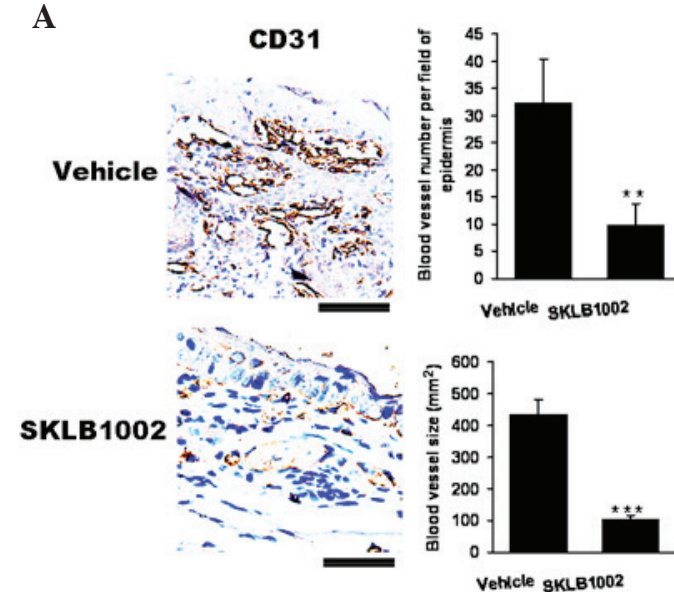

Vehrle SKLB1002

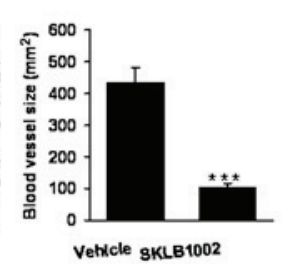

B

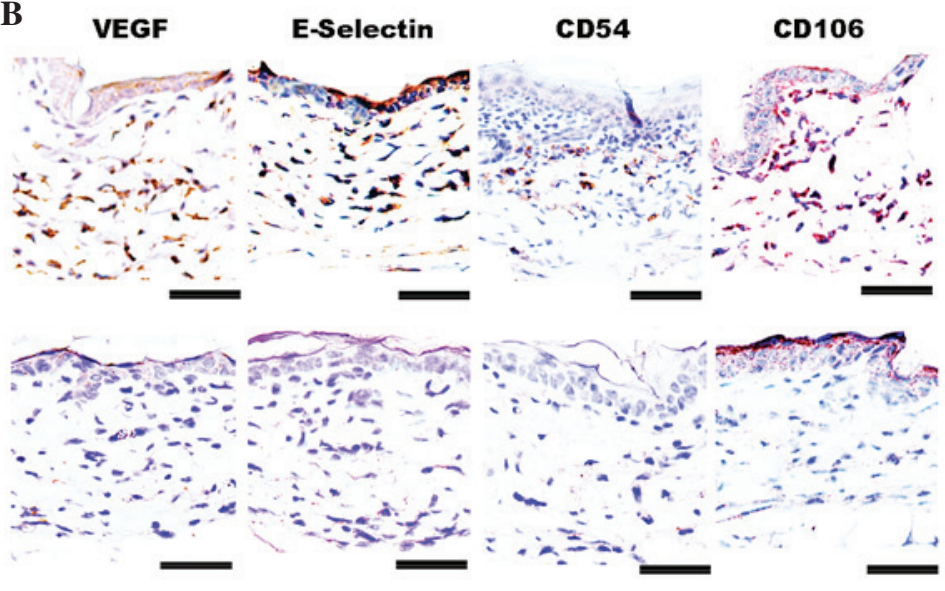

Figure 4. Reduced vascular abnormalities after SKLB1002 treatment. (A) Immunohistochemical staining for CD31 showed increased vascular density and enlargement of blood vessels in the control group. (B) Immunohistochemistry to detect related chemokines and growth factors in SKLB1002-treated mice $(n=5)$ and vehicle-treated mice $(n=5)$. Data are representative of three independent experiments. Data represent the means \pm SD $\left({ }^{* *} \mathrm{P}<0.01,{ }^{* * *} \mathrm{P}<0.001\right)$.

VEGF-dependent pathological angiogenesis normalized the blood vasculature.

\section{Discussion}

Psoriasis is a common inflammatory disease and its underlying pathogenesis is still not fully understood $(20,21)$. Therefore, therapeutic interventions are currently limited and restricted to the treatment of symptoms rather than targeting the mechanisms underlying the disease. In the present study, we investigated the activity of a new small molecule VEGF inhibitor in a psoriasis-like mouse model. The three major components of psoriasis pathogenesis, infiltration of leukocytes, hyperproliferation of epidermal keratinocytes, and occurrence of vascular abnormalities, were markedly improved following treatment with SKLB1002. These findings indicate that therapeutic intervention at the level of the vasculature may be sufficient to reduce the immune-mediated and epidermal components of the disease.

SKLB1002 is a new and selective VEGFR2 inhibitor that potently inhibits VEGFR2 with a half maximal inhibitory concentration $\left(\mathrm{IC}_{50}\right)$ of $32 \mathrm{nmol} / \mathrm{l}(16)$. SKLB1002 significantly inhibits HUVEC proliferation, migration, invasion and tube formation in vitro (16). In this study, vascular abnormalities and secretion of VEGF were reduced by treatment with SKLB1002 in K14/VEGF transgenic mice. Besides its role in angiogenesis, VEGF induces hyperpermeability of blood vessels, leading to tissue edema during inflammation (22-24). Furthermore, chronic overexpression of VEGF in the skin of K14-VEGF transgenic mice promoted leukocyte rolling and adhesion in skin microvessels, most likely resulting from the increased expression of adhesion molecules such as E-selectin, CD106 and CD54 $(18,25)$. Thus, the inhibition of these additional activities of VEGF related to attracting and activating T lymphocytes by systemic treatment with VEGFR inhibitor most likely contributed to the diminished inflammatory cell infiltration observed in the skin.

In conclusion, the new and potent VEGFR2 inhibitor SKLB1002 effectively alleviates skin inflammation or completely cures psoriasis in a mouse model. Mechanistic studies indicated that SKLB1002 treatment significantly decreased the number of blood vessels and reduced the tortuosity of epidermal blood, prevented vascular leakage and T lymphocyte infiltration in skin, which may promote skin inflammation. These findings establish a solid basis for future clinical studies of the new selective VEGFR2 inhibitor for the treatment of psoriasis.

\section{Acknowledgements}

This study was supported by the Veterinary Medicine Discipline Program of Southwest University for Nationalities (2011XWD-S0906), and the Applied Basic Research Program of Sichuan Province (2011JY0033). We would like to thank Dr Sheng-Yong Yang from the State Key Laboratory of Biotherapy and Cancer Center, West China Hospital, West China Medical School, Sichuan University for donating SKLB1002.

\section{References}

1. Christensen TE, Callis KP, Papenfuss J, et al: Observations of psoriasis in the absence of therapeutic intervention identifies two unappreciated morphologic variants, thin-plaque and thick-plaque psoriasis, and their associated phenotypes. J Invest Dermatol 126: 2397-2403, 2006.

2. Bowcock AM and Krueger JG: Getting under the skin: the immunogenetics of psoriasis. Nat Rev Immunol 5: 699-711, 2005.

3. Creamer D, Allen MH, Sousa A, Poston R and Barker JN: Localization of endothelial proliferation and microvascular expansion in active plaque psoriasis. Br J Dermatol 136: 859-865, 1997.

4. Pinkus $\mathrm{H}$ and Mehregan AH: The primary histologic lesion of seborrheic dermatitis and psoriasis. J Invest Dermatol 46: 109-116, 1966

5. Liew SC, Das-Gupta E, Chakravarthi S, et al: Differential expression of the angiogenesis growth factors in psoriasis vulgaris. BMC Res Notes 5: 201, 2012.

6. Detmar M, Brown LF, Claffey KP, et al: Overexpression of vascular permeability factor/vascular endothelial growth factor and its receptors in psoriasis. J Exp Med 180: 1141-1146, 1994.

7. Flisiak I, Zaniewski P, Rogalska M, Mysliwiec H, Jaroszewicz J and Chodynicka B: Effect of psoriasis activity on VEGF and its soluble receptors concentrations in serum and plaque scales. Cytokine 52: 225-229, 2010.

8. Cordiali-Fei P, Trento E, D'Agosto G, et al: Effective therapy with anti-TNF-alpha in patients with psoriatic arthritis is associated with decreased levels of metalloproteinases and angiogenic cytokines in the sera and skin lesions. Ann NY Acad Sci 1110: 578-589, 2007. 
9. Nielsen HJ, Christensen IJ, Svendsen MN, et al: Elevated plasma levels of vascular endothelial growth factor and plasminogen activator inhibitor-1 decrease during improvement of psoriasis. Inflamm Res 51: 563-567, 2002.

10. Detmar M: The role of VEGF and thrombospondins in skin angiogenesis. J Dermatol Sci 24 (Suppl 1): S78-S84, 2000.

11. Yano K, Kajiya K, Ishiwata M, Hong YK, Miyakawa T and Detmar M: Ultraviolet B-induced skin angiogenesis is associated with a switch in the balance of vascular endothelial growth factor and thrombospondin-1 expression. J Invest Dermatol 122: 201-208, 2004.

12. Man XY, Yang XH, Cai SQ, Yao YG and Zheng M: Immunolocalization and expression of vascular endothelial growth factor receptors (VEGFRs) and neuropilins (NRPs) on keratinocytes in human epidermis. Mol Med 12: 127-136, 2006.

13. Elias PM, Arbiser J, Brown BE, et al: Epidermal vascular endothelial growth factor production is required for permeability barrier homeostasis, dermal angiogenesis, and the development of epidermal hyperplasia: implications for the pathogenesis of psoriasis. Am J Pathol 173: 689-699, 2008.

14. Canavese M, Altruda F, Ruzicka T and Schauber J: Vascular endothelial growth factor (VEGF) in the pathogenesis of psoriasis - a possible target for novel therapies? J Dermatol Sci 58: 171-176, 2010.

15. Cao Y: Opinion: emerging mechanisms of tumour lymphangiogenesis and lymphatic metastasis. Nat Rev Cancer 5: 735-743, 2005.

16. Zhang S, Cao Z, Tian H, et al: SKLB1002, a novel potent inhibitor of VEGF receptor 2 signaling, inhibits angiogenesis and tumor growth in vivo. Clin Cancer Res 17: 4439-4450, 2011.

17. Hedlund EM, Yang X, Zhang Y, et al: Tumor cell-derived placental growth factor sensitizes antiangiogenic and antitumor effects of anti-VEGF drugs. Proc Natl Acad Sci 110: 654-659, 2013.
18. Xia YP, Li B, Hylton D, Detmar M, Yancopoulos GD and Rudge JS: Transgenic delivery of VEGF to mouse skin leads to an inflammatory condition resembling human psoriasis. Blood 102: 161-168, 2003.

19. Baker BS, Brent L, Valdimarsson H, et al: Is epidermal cell proliferation in psoriatic skin grafts on nude mice driven by T-cell derived cytokines? Br J Dermatol 126: 105-110, 1992.

20. Lowes MA, Chamian F, Abello MV, et al: Increase in TNF-alpha and inducible nitric oxide synthase-expressing dendritic cells in psoriasis and reduction with efalizumab (anti-CD11a). Proc Natl Acad Sci USA 102: 19057-19062, 2005.

21. Schonthaler HB, Huggenberger R, Wculek SK, Detmar M and Wagner EF: Systemic anti-VEGF treatment strongly reduces skin inflammation in a mouse model of psoriasis. Proc Natl Acad Sci USA 106: 21264-21269, 2009.

22. Ferrara N, Gerber HP and LeCouter J: The biology of VEGF and its receptors. Nat Med 9: 669-676, 2003.

23. Kunstfeld R, Hirakawa S, Hong YK, et al: Induction of cutaneous delayed-type hypersensitivity reactions in VEGF-A transgenic mice results in chronic skin inflammation associated with persistent lymphatic hyperplasia. Blood 104: 1048-1057, 2004.

24. Dvorak HF, Brown LF, Detmar M and Dvorak AM: Vascular permeability factor/vascular endothelial growth factor, microvascular hyperpermeability, and angiogenesis. Am J Pathol 146: 1029-1039, 1995

25. Detmar M, Brown LF, Schon MP, et al: Increased microvascular density and enhanced leukocyte rolling and adhesion in the skin of VEGF transgenic mice. J Invest Dermatol 111: 1-6, 1998. 